\title{
LA COMMUNICATION SOUS FORME D'UN JEU DE PLATEAU POUR PARTAGER DES DONNÉES ET DES RESSENTIS D'EXPERTS À PROPOS D'UN NOUVEAU TRAITEMENT CONTRE LE CANCER
}

\author{
Stéphane Goria ${ }^{1}$, Cléo Boninsegna ${ }^{2}$, Juliette LANDON ${ }^{3}$, \\ Céline Frochot ${ }^{4}$, Claire Vicentini ${ }^{5}$, Camille MuncK ${ }^{6}$, \\ Serge $\mathrm{MORDON}^{7}$, Valentine ANNE ${ }^{8}$
}

La conception de graphiques à des fins de communication ou, de manière plus générale, la visualisation de données est un champ de recherches relativement ancien qui a subi de profonds change-

1 Stéphane Goria est maître de conférences en Sciences de l'information et de la communication au CREM-Université de Lorraine.

2 Cléo Boninsegna est responsable du Knowledge Management pour la valorisation des infrastructures de recherche à l'Université de Lorraine.

3 Juliette Landon est assistante commerciale à L'Atelier NSB - Adegem La Fibre Verte, étudiante de DUT Information-Communication, Communication des Organisations et stagiaire conceptrice au niveau graphique de la représentation inspirée par le jeu.

4 Céline Frochot est directrice de recherches CNRS au Laboratoire Réactions et Génie des Procédés, Université de Lorraine-CNRS UMR 7274.

5 Claire Vicentini est dermatologue à l'hôpital Claude Huriez CHR de Lille et membre de l'équipe OncoTHAI (Thérapies Lasers Assistées par l'Image en Oncologie).

6 Camille Munck est pneumologue et chef de Clinique au CHRU de Lille et membre de l'équipe OncoTHAI (Thérapies Lasers Assistées par l'Image en Oncologie).

7 Serge Mordon est biophysicien, directeur de Recherche INSERM : directeur de l'unité INSERM 1189 OncoThAI (Thérapies Lasers Assistées par l'Image en Oncologie), CHU de Lille Université de Lille.

8 Valentine Anne est docteure en chirurgie thoracique et cardiovasculaire, Hôpitaux Privés de Metz. 
ments au cours de ces dernières années. Or, parmi les différentes possibilités de représentations offertes, celles inspirées par le jeu forment une tendance relativement nouvelle dont les avantages peuvent être nombreux. Ce travail traite de cette forme particulière de communication en présentant le cas d'un support informationnel rappelant un jeu de stratégie sur plateau développé dans un but informatif à propos d'un nouveau procédé de lutte contre le cancer : la thérapie photodynamique.

La conception de graphiques à des fins de communication ou, de manière plus générale, la visualisation de données ${ }^{9}$ est un champ de recherche relativement ancien qui a subi de profonds changements, notamment au cours du $\mathrm{Xx}^{\mathrm{e}}$ siècle, et qui continue d'évoluer (Plantin, 2014 ; Ward et al., 2015 ; Kirk 2016). Malgré ces évolutions, la présentation de données scientifiques destinées à des communautés d'experts, dans un objectif de comparaison de résultats, se contente, en général, de représentations graphiques prenant la forme de diagrammes devenus des classiques en statistique (courbes, histogrammes, camemberts, radars, nuages de points, boîtes à moustaches ou matrices de nuages de points). De même, si le jeu a fait l'objet de nombreuses recherches au cours de ce même siècle (Caillois, 1958 ; Huizinga, 1951 ; Henriot, 1989 ; Yonnet, 1999), depuis une vingtaine d'années, il est aussi envisagé en tant que support informationnel pour traiter et communiquer des données (Grund \& Schelkle, 2016 ; Ryan, 2016). Le jeu est désormais aussi considéré comme un outil permettant la réalisation de tâches professionnelles (on parle alors de serious game ou de jeu sérieux) (Abt, 1970 ; Alvarez \& Djaouti, 2012) ou bien comme une source d'inspiration pour la conception ou mise en œuvre d'outils ou de services professionnels (on parle dans ce cas plutôt de gamification ou de ludification) (Kapp et al., 2014; Zichermann \& Linder, 2013).

Malgré une augmentation constante du nombre de recherches consacrées au jeu ou à la visualisation de données, nous constatons

9 Les données ici sont comprises dans une acception large, c'est-à-dire en tant qu'unité minimale de construction d'information résultant le plus souvent d'une mesure ou d'une estimation (Le Béchec, 2016). 
actuellement qu'en dehors des applications destinées à l'apprentissage, les travaux qui s'intéressent à la question de la communication d'informations scientifiques et techniques à partir de jeux ou inspirées par le jeu sont rares. Nos recherches s'inscrivent en ce sens et portent sur le développement d'un outil de communication qui n'est pas destiné à former, mais à informer notamment des experts dans un contexte d'innovation médicale. Ce n'est pas un jeu, mais un objet qui en prend la forme. Ainsi, nous présentons dans cet article la manière dont un artéfact rappelant un jeu de plateau a été conçu et comment il peut être employé à des fins de mise en évidence et de communication de données et de points de vue d'experts. Nous supposons donc qu'il y a un intérêt à présenter autrement des données et que l'originalité permise par une représentation évoquant le jeu pour faire attirer l'attention, permet aussi de générer des discussions et de faire émerger des connaissances implicites. Nous verrons aussi que cet artéfact, une fois élaboré, peut aussi servir d'intermédiaire pour collecter de nouvelles données.

Ainsi, en élaborant un graphique de données rappelant une partie de jeu sur plateau, nous posons la question de l'utilité de ce type d'outil pour communiquer ou collecter des données auprès d'un public expert. Mais, pour y parvenir, nous donnons d'abord des pistes de réponse à quatre questions :

- Comment élaborer une visualisation de données sous une forme rappelant un jeu de plateau pour communiquer des données scientifiques?

- Y retrouve-t-on des éléments relevant du jeu ?

- Comment ce type de média est-il perçu par le public auquel il est destiné ?

- Peut-on employer cet outil à d'autres fins ?

Avant de présenter les réponses à ces questions, nous introduisons et décrivons brièvement le concept de jeu. Nous définissons et analysons ses caractéristiques structurelles et présentons une échelle qui permet de positionner notre représentation par rapport au jeu. Nous expliquons ensuite la méthode de conception utilisée pour développer le jeu de plateau. Enfin, nous abordons 
la phase de réception de cette visualisation physique de données auprès du public pour lequel elle a été conçue.

\section{Le jeu, la gamification et le disengamement}

Il existe au moins deux manières de qualifier le jeu. La première consiste à raisonner en termes d'activité de jeu, ce que l'on nomme «play » en anglais. Roger Caillois (1958, pp. 42-43), à la suite des travaux de Johan Huizinga (1938), a considéré qu'une véritable activité de jeu se devait de respecter six critères : être (1) libre, (2) séparée et identifiable en termes d'espace et de temps, (3) incertaine, (4) improductive, (5) soumise à des règles et (6) fictive. Toutefois, si l'on souhaite employer le jeu à des fins sérieuses comme en éducation, cette définition du jeu pose problème, car elle exclut, de fait, ce qui ressemble à un jeu à partir du moment où le « jeu » mène à une production de connaissances et qu'en contexte éducatif l'apprenant est plus souvent contraint que libre de jouer. Pour pallier ces problèmes, une autre approche consiste à qualifier un jeu à partir de la structure qui sert à jouer, ce que les anglophones nomment «game ». Dans la littérature scientifique francophone, c'est Jacques Henriot qui, le premier, a proposé, dans une analyse des différents aspects du jeu (structure, activité et attitude) de qualifier de jeu ce qui, pour un observateur extérieur ou pour le joueur, ressemble à un autre jeu (Henriot, 1969, p. 19). Sur ce principe, des concepteurs de jeu comme Jesse Schell (2010, p. 51) ou Katie Salen \& Eric Zimmerman (2004, p. 80) ont proposé des définitions du jeu à partir de six composants essentiels :

(1) L'esthétique (qui rend compte des relations établies à partir d'un ou plusieurs des cinq sens entre le jeu et le joueur);

(2) Les mécanismes (qui regroupent les procédures et les règles qui sont au cœur du jeu) ;

(3) La technologie (qui fait le bilan de tous les matériels et matériaux qui rendent le jeu possible) ;

(4) L'histoire (qui propose un univers fictionnel pour projeter le joueur dans un monde imaginaire, un ensemble de séquences d'événements); 
(5) Le challenge (qui correspond à un ou plusieurs objectifs que doit atteindre le joueur pour gagner au sens où le jeu lui permet d'aboutir à une situation finale satisfaisante et différente de la situation initiale de début du jeu);

(6) L'expérience des possibles (qui rassemble les possibilités offertes au joueur à travers les mécanismes du jeu « perdre » ou " gagner », même s'il ne peut y avoir qu'une seule façon de gagner ou de perdre).

Les quatre premiers éléments correspondent à la tétrade de Jesse Schell. Cette tétrade est très pratique, mais peut aussi paraître trop limitée pour déterminer ce qui est à considérer ou non comme un jeu. Par exemple, le composant histoire d'un jeu de type puzzle, casse-tête ou un jeu de construction n'est pas toujours véritablement présent. Dans de nombreux cas, c'est le joueur qui crée sa propre histoire à partir du jeu. S'il joue, c'est aussi parce qu'un challenge récréatif lui est proposé. Jesse Schell considère d'ailleurs le challenge comme étant au centre du design de tous les jeux (Schell, 2010, p. 133) et le fait d'intégrer le challenge comme composant d'une structure de jeu permet de distinguer les jeux des jouets. Ainsi, J. Alvarez et D. Djaouti (2012, p. 11) proposent de distinguer les jouets des jeux par le fait que l'usage «normal » d'un jouet n'est pas associé à l'atteinte d'un objectif explicite donné au joueur. Enfin, un autre composant important pour définir, a minima, un jeu est celui de la proposition d'une expérience des possibles (situations d'échecs, de réussite, d'exploration, etc.) (Genvo, 2013, p. 47 ; Salen \& Zimmerman, 2004, p. 67). Il en effet difficile de qualifier une chose de jeu si celle-ci ne permet pas cette expérience des possibles.

Utiliser la composition d'une structure de jeu «standard» pour analyser des artéfacts et évaluer leur proximité avec le jeu nous semble plus pratique et moins exclusif que de définir un jeu en tant qu'activité. Cela nous donne l'opportunité de considérer un continuum séparant les structures ludiques des structures non ludiques (Figure 1). 


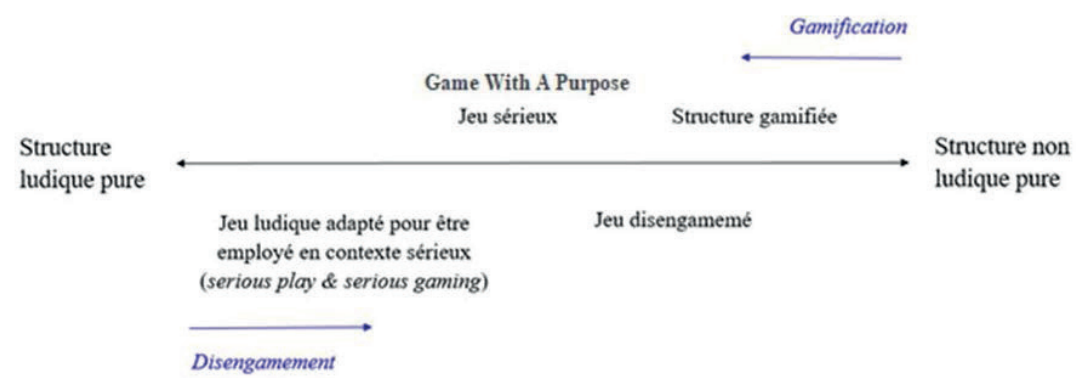

Figure 1. Continuum liant les jeux ludiques à des structures plus sérieuses

La figure 1 met en évidence deux modes de raisonnement opposés pour concevoir des structures plus ou moins ludiques afin d'atteindre un but sérieux. Elle présente un axe principal qui peut être parcouru de la droite vers la gauche en considérant un processus de conception faisant appel à un processus de gamification (d'ajouts d'éléments qui font ou rappellent le jeu à une structure non ludique) et de la gauche vers la droite par un processus de disengamement (processus de déformation et de retraits d'éléments d'un jeu ludique pour en faire un outil sérieux).

Dans le sens de la gamification, il est surtout question de résoudre des problèmes d'intérêt, d'attractivité ou d'engagement de personnes pour exploiter ou mieux s'approprier une structure non ludique préexistante. La solution à apporter passe, dans ce cas, par une série de modifications de la structure non ludique consistant en l'ajout de nombreux éléments issus du design de jeux (Burke, 2014, p. 16, Huotari \& Hamari, 2017). La structure n'atteint pas forcément un stade de développement suffisant pour lui permettre d'être assimilable à un jeu (Deterding et al., 2014). Le continuum de cet axe est aussi là pour montrer qu'il existe différents degrés de gamification des structures qui à l'extrême donne naissance à de véritables jeux. Dans le sens du disengamement, la résolution d'un problème ne prend pas en compte une base non ludique préexistante, mais va consister à rechercher un jeu dont certains aspects (en général plutôt mécaniques ou esthétiques) seront identifiés comme des réponses intéressantes au problème considéré (Goria, 
2016). Par rapport à un processus de résolution de problème par gamification, lors d'une conception par disengamement, ce ne sont pas des éléments de conception de jeux (game design patterns) (Morschheuser et al., 2017) qui sont recherchés pour apporter une réponse à un problème, mais un jeu spécifique qui sera détourné et déformé pour apporter une réponse. Lorsque les modifications à apporter sont relativement faibles, ce type de détournement de jeu peut être assimilé à la mise en œuvre d'activités de serious play et de serious gaming $^{10}$, puisque dans ces cas, la structure de jeu n'a été que légèrement altérée (Alvarez \& Djaouti, 2012, p. 12). Lorsque ces modifications sont très importantes (par exemple, quand le jeu n'a été utilisé seulement comme source d'inspiration d'une certaine esthétique), nous sommes dans le cas extrême des jeux disengamemés (par exemple, l'entreprise Brave Space Design propose des étagères Tetrad Flat directement inspirées du jeu vidéo Tetris). L'intérêt de l'approche par disengament tient dans la manière dont un problème est abordé. Une fois l'énoncé du problème compris, il s'agit de rechercher le jeu le plus adéquat pour y répondre pour ensuite, le détourner, le déformer et l'adapter pour apporter une réponse au moins partielle au problème. Pour terminer ce propos, sur l'axe de la figure 1, le jeu disengamemé et la structure gamifiée ne sont pas placées exactement l'un sous l'autre, car, de manière générale nous supposons qu'un jeu, même fortement disengamemé, rappellera plus facilement le jeu qu'une structure peu gamifiée.

Plus ou moins au centre de l'axe de la figure 1, se situent des structures plus difficiles à interpréter (sans informations à propos de l'histoire de leur conception) en termes de processus de gamification ou de disengamement. Nous y retrouvons d'abord les structures ludiques « sérieuses » incluant une distinction entre les

10 L'expression "serious play » signifie qu'un jeu ou un jouet (comme les briques Lego(C) est associé à une pratique sérieuse sans que sa structure n'en soit fondamentalement affectée, même si quelques modifications peuvent avoir été faites. Par exemple, la pratique en cours d'Histoire du jeu vidéo Assassin's Creed Origins (Ubisoft), dont la version Discovery tour est dédiée à cette pratique, relèvera du serious gaming. Ceci dit, l'expression « serious gaming » est employée par la plupart des auteurs francophones comme synonyme de celle de « serious play», mais elle peut aussi se référer à la pratique continue de serious games. 
« jeux sérieux » et les « jeux à but » ou GWAP (pour Game With A Purpose) (Lafourcade, 2015). Dans le cas des « jeux sérieux », le « joueur » bénéficie de la solution apportée au problème. Dans le cas des GWAP, le " joueur » contribue à la résolution d'un problème qui ne le concerne pas forcément directement, mais dont l'aspect ludique l'incite à y participer ${ }^{11}$. Ceci dit, pour certains GWAP la structure ludique est discutable, laissant plutôt place à une structure plus « sympathique » que ludique visant une collecte de données par un grand nombre de personnes. C'est par exemple le cas de Zooniverse ${ }^{12}$ qui est classé parmi les GWAP, mais qui est plus difficilement assimilable à un jeu.

\section{La visualisation de données sous la forme d'un jeu de plateau}

Le problème de visualisation de données à résoudre portait sur la mise en évidence des avantages de deux types de traitements du cancer selon la perception d'un groupe d'experts. Nous avons fait l'hypothèse qu'une visualisation de données scientifiques sous la forme d'un jeu de plateau pourrait compléter des supports de communication plus traditionnels. Ce projet prend place dans le cadre d'une recherche-action dont l'objectif est de participer à la promotion d'un procédé de lutte contre le cancer dit " thérapie photodynamique ", abrégée PDT pour PhotoDynamic Therapy. Cette thérapie utilise une molécule et de la lumière. Après activation par la lumière, la molécule est capable de transférer l'énergie à l'oxygène du milieu environnant pour produire des espèces réactives de l'oxygène qui vont détruire les tissus ciblés (Frochot et al., 2007). Du point de vue du « jeu », cette recherche consiste à comprendre l'apport potentiel d'un artéfact au sens de P. Rabardel (1995), c'est-à-dire d'une « chose susceptible d'un usage, élaborée pour s'inscrire dans des activités finalisées [...] [qui] concrétise une solution à un problème ou à une classe de problèmes sociale-

11 Pour qu'un joueur tire un bénéfice direct d'un Gwap, il lui faut disposer d'un statut lui donnant accès rapidement à l'évolution des données collectées ou être alerté dès qu'une solution à problème qui l'intéresse a été enregistrée par le biais du GWAP.

$12 \mathrm{https}: / /$ www.zooniverse.org 
ment posés » (Rabardel, 1995, p. 56). Dans le contexte qui nous intéresse, il s'agit d'un artéfact évoquant le jeu destiné à promouvoir et discuter la PDT avec des spécialistes de cette thérapie, mais aussi d'autres experts et chercheurs intéressés par le traitement du cancer. Pour ce faire, une forme de cancer a été choisie pour cette représentation : celle des lésions précancéreuses de la peau appelées Kératoses Actiniques (dues généralement à une exposition solaire intense, prolongée et répétée). Les deux traitements représentés ont été le traitement de type PDT et un traitement médicamenteux à base de crème ou de gel dit « topique ».

Pour débuter la conception d'une visualisation de données sous forme de jeu, qui avait pour but de comparer les deux traitements, nous avons employé un processus de disengamement et nous nous sommes inspirés d'un jeu de guerre (SPQR, GMT Games, 1992) dont nous allons expliciter les grandes étapes. Il a d'abord fallu réaliser une analogie entre les deux armées du jeu originel et les deux types de traitements du cancer. Puis, il a été nécessaire d'imaginer la forme que prendrait l'affrontement entre ces deux traitements. Nous avons privilégié une lutte sur un plateau entre deux armées qui, au départ, se font face de part et d'autre d'une rivière. Celle-ci peut être traversée et sert de ligne de démarcation avant le déclenchement de la « bataille». Une fois la bataille déclenchée, il y a des zones du plateau où une armée est localement supérieure à l'autre, tandis que, dans d'autres zones, les unités des armées se neutralisent, voire ne s'affrontent pas (il peut y avoir des espaces vides).

Pour obtenir une représentation permettant la mise en évidence de telles situations, il faut être capable de déterminer à quoi correspond un pion d'une armée du jeu originel ; c'est-à-dire comment le représenter et le placer sur le plateau. Pour faire simple, chacun des deux traitements s'est d'abord vu attribuer une couleur spécifique : blanc ou noir. Le positionnement des pions est réalisé à partir d'une décomposition d'un parcours d'expériences du patient atteint de kératoses actiniques. L'emploi d'un parcours d'expériences afin d'aider à représenter l'expérience utilisateur sous la forme d'un corridor (Kim \& Mauborgne, 2000) ou d'une carte fait désormais partie des outils classiques du développement 
informatique (Daumal, 2015) et du design thinking (Kelley \& Kelley, 2016, p. 196). Après plusieurs discussions et réflexions, le nombre d'étapes de ce parcours a été réduit à quatre pour permettre une plus forte lisibilité des éléments présentés sur le futur plateau de jeu. Les étapes qui ont été retenues ou regroupées sont : (1) Être informé de l'existence de la thérapie, la connaître et être convaincu de son utilité, (2) Formation du personnel et mise en place des équipements des éléments nécessaires à la mise en œuvre de la thérapie, (3) Utilisation des matériels et traitement du patient, (4) Phase de Récupération du patient à son domicile.

Pour compléter ce parcours, une liste d'attributs inspirés des leviers d'utilité pour l'usager (Kim \& Mauborgne, 2000) a été établie. Les attributs retenus devaient être considérés comme des critères importants, sachant qu'ils serviraient à l'évaluation, sur une échelle de 0 à 4, étape par étape, du parcours d'expérience patient des deux types de thérapies. Ce parcours et cette liste d'attributs ont ensuite été soumis à des experts de la PDT afin de corriger ou reformuler certaines étapes ou certains attributs. Au final, les attributs suivants ont été retenus :

(1) Adaptabilité en fonction du changement du cadre d'application,

(2) Sensation de bien-être a priori et image positive du traitement,

(3) Coûts (financiers, temporels, humains, ...),

(4) Gain de productivité,

(5) Perception atténuée des risques induits,

(6) Émotion positive (ressenti de plaisir ou atténuation d'une douleur),

(7) Simplicité d'emploi du traitement,

(8) Variété des choix proposés.

Une fois les étapes et les attributs validés, ils ont d'abord été croisés sous la forme d'une matrice comme le proposent W.C. Kim et R. Mauborgne avec leur Buyer Utility Map (Kim \& Mauborgne, 2000) (voir figure 2). Or, plutôt que d'estimer directement, pour chaque case de la matrice, les points forts ou faibles de l'un ou l'autre des traitements, ce sont des questions qui ont été formu- 
lées. Chaque case ne doit pas forcément accueillir une question et certaines cases peuvent éventuellement en accueillir plusieurs. Chacune de ces questions correspond ainsi à une interprétation de la combinaison d'un attribut et d'une étape et formulée en fonction du contexte de l'étude. Ce qui doit amener la personne interrogée à donner une réponse sous la forme d'un nombre entier sur une échelle de 0 à 4 (4 étant la meilleure note). À chaque fois, les deux thérapies (PDT et traitement topique) sont questionnées.

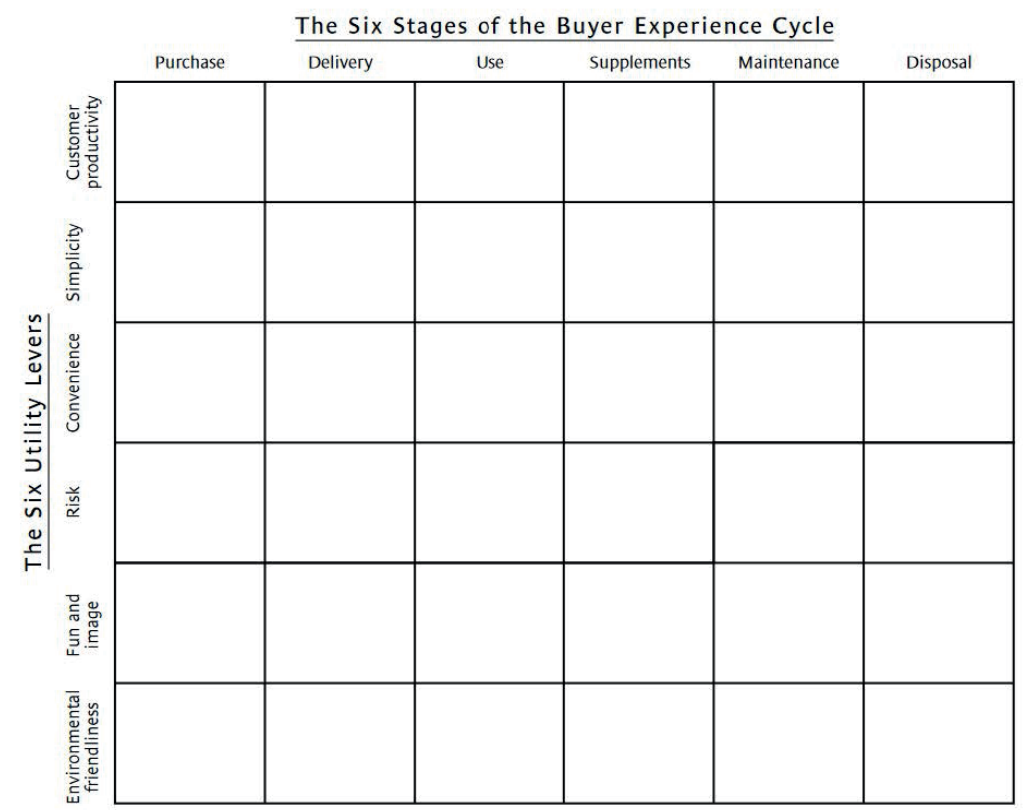

Figure 2. Buyer Utility Map (Kim \& Mauborgne, 2000)

Par exemple, pour la case qui correspond à l'intersection de la troisième étape, « utilisation des matériels et traitement du patient » et du sixième attribut « émotion positive (ressenti de plaisir ou atténuation d'une douleur) », nous avons formulé les deux questions suivantes :

- Diriez-vous qu'il y a une atténuation de la douleur grâce au traitement topique et, si tel est le cas, à quelle hauteur sur une échelle de 0 à 4 ( 0 aucune atténuation -4 très forte atténuation)? 
- Diriez-vous qu'il y a une atténuation de la douleur grâce à la PDT et, si tel est le cas, à quelle hauteur sur une échelle de 0 à 4 ( 0 aucune atténuation -4 très forte atténuation) ?

De même, pour la quatrième étape " Phase de récupération du patient à son domicile » et le premier attribut « Adaptabilité en fonction du changement du cadre d'application ", nous avons formulé les deux questions suivantes :

- Sur une échelle de 0 à 4 , pensez-vous que le traitement topique est bien adapté au cadre de vie du patient une fois en phase de récupération à son domicile ? ( 0 pas du tout, 4 très bien adapté).

- Sur une échelle de 0 à 4, pensez-vous que la PDT est bien adaptée au cadre de vie du patient une fois en phase de récupération à son domicile ? (0 pas du tout, 4 très bien adapté).

Ces questions ont ensuite été regroupées pour former un questionnaire qui a fait encore l'objet de reformulations suite à des tests avec deux experts connaissant au moins l'une ou l'autre des deux thérapies. Alors seulement, le questionnaire a été posté à un ensemble d'experts des deux thérapies. Huit experts nous ont ainsi répondu et chacune des réponses a ensuite été associée à une note moyenne arrondie à l'entier supérieur. Ainsi, les réponses obtenues permettaient un positionnement sur un plateau de jeu.

\section{Mise en place du plateau et des pions}

Le plateau de jeu a été divisé en quatre larges colonnes, identifiables chacune par un château correspondant aux étapes précédemment retenues. Sous ces châteaux, nous retrouvons six colonnes qui vont servir pour placer les pions. Ces derniers ont été fabriqués à l'aide d'une imprimante 3D et entourés d'une ceinture de couleur associée à un attribut signalé par une légende (voir figure 3). Le placement des pions a été effectué en fonction des notes sur une échelle inverse de celle employée dans le questionnaire afin d'obtenir la représentation suivante : plus un pion d'un 
traitement est proche de la rivière et plus l'aspect du traitement auquel il fait référence est apprécié. Si une question obtient la note de 0 , aucun pion n'est posé pour le camp auquel cette question est liée. Si une question obtient la note de 4 en réponse, un pion à la couleur du camp et de l'attribut est placé dans la colonne de l'étape concernée sur la ligne 1 de son camp (à une case de la rivière). Pour obtenir cet emplacement, le calcul suivant est fait : 5 - note obtenue en réponse à la question (ce calcul n'est valable que si la note est différente de 0 ).

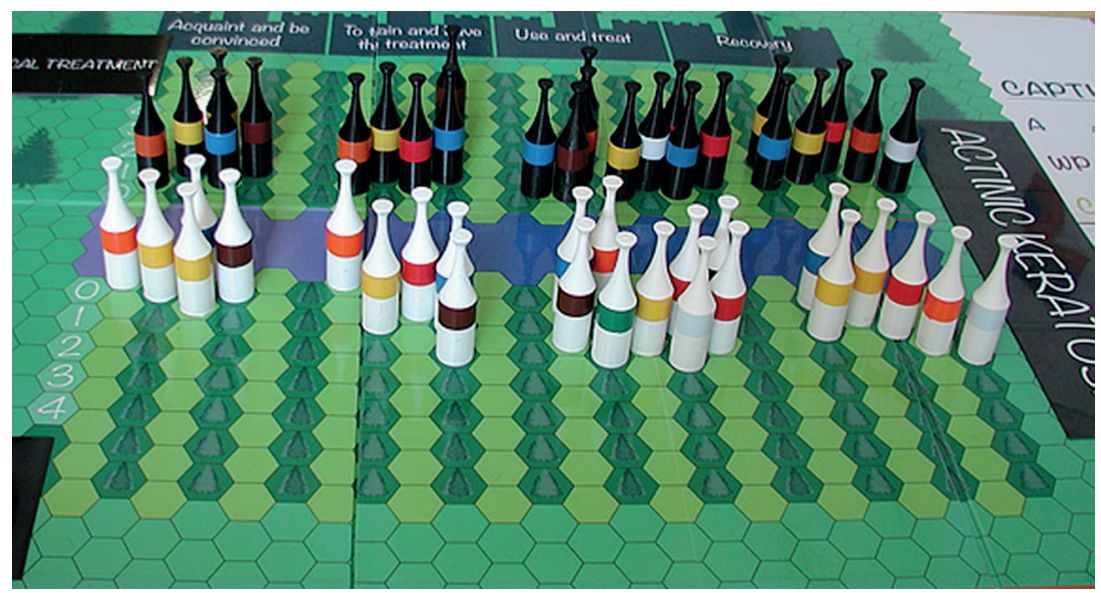

Figure 3. Présentation " perception 》 des rapports de forces, après déplacement, entre le traitement topique (pions noirs) et la PDT (pions blancs)

Ceci fait, la présentation peut être modifiée afin de rendre compte, soit uniquement des déséquilibres entre les forces dans une version « perception », soit opter pour une présentation " perception \& problème » permettant le signalement de certains problèmes. Dans la version «perception », la phase d'estimation des rapports de forces en présence, colonne par colonne est effectuée directement. S'il y a égalité ou si la différence entre les deux camps est localement (pour la colonne concernée) faible, rien n'est à changer. Si la différence est plus notable (d'au moins $10 \%$ ), il faut avancer d'une case tous les pions du camp supérieur et faire reculer d'une case les pions du camp inférieur. Si la différence est 
plus importante (d'au moins $25 \%$ ), le déplacement effectué est de deux cases et, s'il est vraiment très important (d'au moins $50 \%$ ), de 3 cases. De la sorte, une représentation qui rappelle une partie de jeu est obtenue (Figure 3).

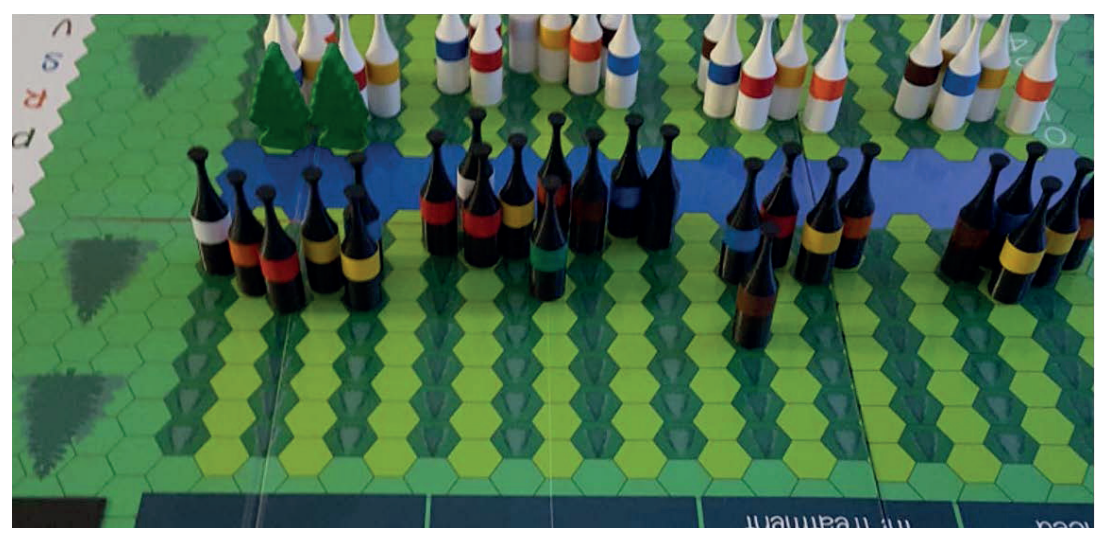

Figure 4. Présentation en mode "perception \& problèmes 》 du traitement Topique (pions noirs) face à la PDT dont les pions (blancs) sont bloqués par des sapins (en haut à gauche)

En mode de présentation « perception \& problèmes », un ajout d'informations, colonne par colonne, est effectué. Ce changement est matérialisé par l'ajout d'une autre catégorie de pions. Il s'agit de la mise en évidence de problèmes particuliers rencontrés et qui sont mal mis en évidence par les attributs (les variables) précédemment sélectionnés. Pour ce faire, un pion en forme de sapin sert à représenter un obstacle supplémentaire à franchir et qui ne peut se rencontrer que d'un côté du plateau (un seul camp). Ces obstacles sont placés sur la ligne 0 d'un camp (qui se situe de chaque côté de la rivière). Si le problème est signalé pour un seul traitement. Si le problème se retrouve dans les deux traitements, un obstacle est placé en miroir de part et d'autre de la rivière. Ensuite, le calcul des mouvements des autres pions peut se faire comme indiqué ci-dessus, à la différence près que chaque obstacle (représenté par un sapin) réduit les mouvements d'une case des pions du camp et de la colonne sur lesquels il se situe. Il faut donc une différence d'au moins $25 \%$ pour que des pions progressent d'une seule case 
s'il y a un sapin dans cette colonne du côté de ce camp. Pour une même colonne, un sapin signale un problème jugé important et deux sapins signalent soit un problème jugé très important, soit deux problèmes jugés importants (Figure 4).

Dans notre cas d'exemple, le sapin posé dans la dernière colonne (récupération) rend compte d'une différence notable entre l'essai clinique et la mise en pratique en contexte réel de la PDT lors de la phase de récupération du patient. Si nous prenons le cas du traitement topique par rapport à la PDT, tant du point de vue des experts (Figure 3) que des essais cliniques (Togsverd et al., 2017), la PDT semble supérieure au traitement topique en termes de résultats obtenus après un ou deux traitements. Cependant, en situation de traitement hors essais cliniques, des problèmes peuvent se poser. Par exemple, dans le cas de la PDT, après les premiers jours de traitement dermatologique au niveau du visage, si le patient suit bien les instructions, il subit une forte inflammation et des rougeurs durant quelques jours. Ensuite seulement, ce désagrément esthétique s'estompe. Or, en dehors du cas de l'essai clinique où une pression et un suivi plus importants sont exercés sur le patient, l'apparition de fortes rougeurs et d'inflammations risque d'amener le patient à arrêter le traitement afin de retrouver plus vite un visage « normal ». L'importance de l'esthétique à très court terme (de quelques jours à une semaine) est alors bien plus importante que l'obtention de résultats durables après un délai un peu plus long (un à trois mois). Sur le plateau de jeu, la présence du sapin alerte sur l'existence de ce problème, mais sa nature et sa description ne peuvent pas, pour le moment, figurer sous une forme plus explicite que le commentaire.

\section{La première étude de réception et quelques éléments de conclusion}

À l'occasion d'une conférence internationale dédiée à la PDT : Photodynamic Therapy and Photodiagnosis update 2016, nous avons réalisé une première étude de réception de cette forme de représentation de données par un public intéressé par la PDT dont plusieurs experts qui présentaient leurs travaux concernant 
cette thérapie. Durant une journée complète d'octobre 2016, de 9 à 19 heures, le plateau de jeu et ses pièces (placées conformément aux données récoltées) ont été mis en place pour être présentés dans un espace de la bibliothèque spécialement aménagé pour y présenter les posters des intervenants. Il est à noter qu'il était nécessaire de traverser cet espace pour accéder à la partie restauration de la conférence.

Selon le moment, le « jeu » pouvait être présenté par une personne de sexe masculin ou féminin. L'hypothèse d'une influence du genre de la personne présentant le « jeu » sur le public a été brièvement envisagée. Ce n'était pas l'objet de cette observation, mais une hypothèse qui a émergé lors de la présentation du « jeu ». Le plateau était positionné de manière traditionnelle dans un cadre de jeu, c'est-à-dire sur une table de sorte qu'un joueur puisse se tenir de chaque côté de la table. L'un des côtés était occupé par une personne assise pouvant présenter le « jeu », ses caractéristiques et clarifier les données ainsi présentées, tandis que de l'autre côté la place était libre avec une chaise à proximité afin d'inviter des personnes à s'y installer tout en permettant à des personnes de rester debout et toucher les pièces du jeu.

Nous avons pu ainsi mettre en place trois variantes du protocole :

- Être assis devant le plateau en attendant qu'une personne passe pour l'inviter à « jouer »,

- Être debout à proximité de la table et prêt à répondre à des questions,

- Être suffisamment éloigné de la table pour laisser le «jeu» sans présentateur et voir si des personnes s'en approchaient.

À la fin de cette journée, nous avons pu faire plusieurs observations qui restent limitées à ce cas d'étude, à un nombre restreint d'observés et des conditions d'observation assez sommaires. Nous avons constaté que, pour un public mixte composé d'environ 80 personnes qui ne connaissaient pas le « jeu », 19 personnes se sont approchées du « jeu » suffisamment près pour pouvoir toucher des pièces. Sur ces 19 personnes, 13 hommes et trois femmes 
ont posé des questions à propos du « jeu » lorsqu'il y avait un " présentateur ». Parmi celles-ci, sept hommes et deux femmes ont pris des pions du « jeu » en main. La présentation sous forme de jeu n'a pas choqué les personnes l'ayant vue ; du moins nous n'avons eu aucune remarque, ni identifié une attitude qui laisserait croire à ce genre de réaction. Ce type de présentation a bien permis de discuter des points forts et des points faibles de la PDT concernant son application pour le traitement des kératoses actiniques. Deux hommes ont accepté de « jouer », c'est-à-dire de participer dynamiquement à la collecte de données en leur posant la série de questions préétablies. Ils y ont répondu directement en plaçant les pions sur les cases (une fois les principes de fonctionnement de cette représentation bien compris) en partant de l'estimation du groupe d'experts préalablement interrogé. Ils n'estimaient donc plus les réponses aux questions de leur seul point de vue et prenaient en compte les changements de rapports de forces engendrés sur le plateau. Cela nous a permis, dans le même temps, de constater leur différence de perception des rapports de forces entre les deux traitements. Avec ces derniers, de nouveaux mouvements de pièces ont aussi été envisagés en employant un raisonnement du type « et si ... ». Même si ce retour est très limité, il montre toutefois la possibilité qui existe de co-construire une représentation de ce type à partir d'un affichage correspondant à la perception moyenne d'un petit groupe de personnes.

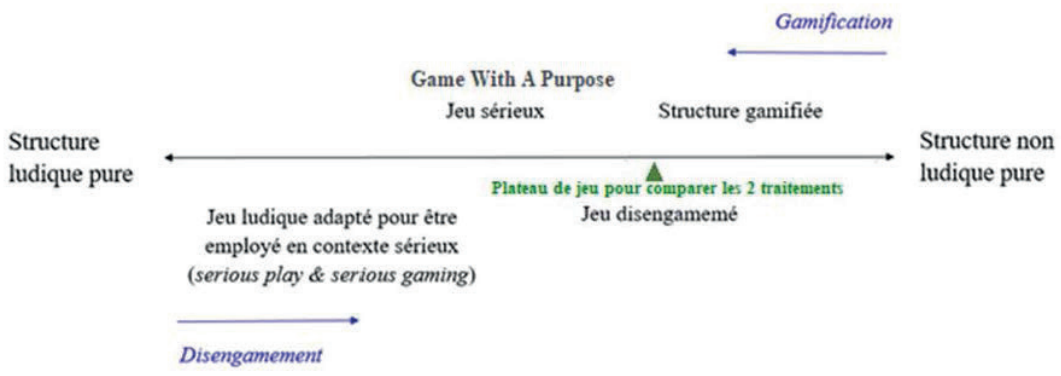

Figure 5. Place de notre représentation sous forme de jeu de plateau sur le continuum liant les jeux ludiques à des structures plus sérieuses 
Finalement, lorsque 1'on considère cette présentation de données sous la forme d'un jeu de plateau, nous nous rendons compte qu'elle se situe sur l'axe de la Figure 1, c'est plutôt une structure gamifiée, mais qui tend légèrement vers les GWAP les moins ludiques (Figure 5).

Le fait qu'une représentation rappelant un jeu puisse servir d'artéfact intermédiaire pour une collecte de données conforte notre avis de la positionner non loin d'un GWAP" ${ }^{13}$. Si les " joueurs » peuvent tirer quelques bénéfices de l'utilisation de cette représentation $^{14}$, les principaux bénéficiaires de la collecte de données restent les membres de l'équipe de recherche (PDT Team) pour laquelle la représentation sous forme de jeu a été conçue. Même si elle ne peut pas être considérée comme un jeu puisqu'elle n'est pas faite pour jouer, si nous reprenons les éléments évoqués plus haut caractérisant une structure de jeu, cette représentation de données en est très proche. Elle dispose bien d'une esthétique et de mécanismes régulant le placement des pièces et leur mouvement, ainsi que d'une technologie puisqu'il y a un support de données formé par les pions et le plateau. Elle comprend aussi une histoire suggérée par les ordres de bataille des pions. Elle permet de faire le lien avec la perception de deux traitements proposés sous la forme d'une séquence avec un début et une fin. Elle contient en outre un challenge incitant les " joueurs » à imaginer comment mieux mettre en valeur la PDT, du moins au niveau de la perception de certaines personnes pour les convaincre de son intérêt. Elle permet in fine une certaine expérience des possibles à partir du raisonnement « et si ... » qui invite les personnes interrogées à imaginer différents futurs. Ainsi, au sens de Jacques Henriot,

13 Il est probable que d'autres personnes déplaceront le curseur (le triangle vert) pour le mettre sous la structure gamifiée. Peut-être que d'autres, le rapprocheront plus encore des GWAP et jeux sérieux. L'utilité de cette échelle de positionnement n'a pas encore été testée pour comparer ces différentes appréciations.

14 Cela peut être aussi le cas, par exemple, avec des étudiants en biochimie qui jouent à Foldit (voir par exemple la vidéo de présentation suivante : https://www.youtube. $\mathrm{com} /$ watch? $\mathrm{v}=\mathrm{WVWXB} 94 \mathrm{hhPM}$ ) et complètent ainsi leur compréhension des structures protéiques. Ceci dit Foldit n'est pas conçu dans ce but, mais pour trouver de nouvelles solutions de pliages permettant de lutter contre des virus du type VIH. 
une personne peut la considérer ou non comme un jeu selon ses a priori sur ce que doit être ou non un jeu et la position qu'elle adopte pour le considérer (en utilisant, c'est-à-dire en tentant d'y " jouer », en se plaçant à une distance réduite permettant de toucher les pièces du « jeu » ou les « joueurs » ou à une distance plus grande, mais permettant de distinguer encore les pièces du « jeu » et le comportement des « joueurs »).

Ce n'est peut-être qu'un jouet et ce qui se passe autour serait du serious play. En effet la proximité de raisonnement avec des approches de réflexion à base, par exemple, de wargames ou de jeu de construction (du genre Lego serious play) détournés de leur usage premier pose question. En ce sens, nous développons actuellement une nouvelle structure inspirée par le jeu et destinée à promouvoir une nouvelle forme de diagnostic médical. Le public ciblé étant différent, nous tenterons de conforter les observations rapportées dans cet article tout en questionnant sa perception du système de représentation proposé en tant que jouet, jeu ou graphique tactile.

\section{Références}

Abt, C. (1970). Serious Games. New York : University Press of America.

Alvarez, J. \& Djaouti, D. (2012). Introduction au Serious game. Paris : Éd. Questions Théoriques.

Burke, B. (2014). Gamify: How gamification motivates people to do extraordinary things, Stamford CT : Gartner INC.

Caillois, R. (1958). Les jeux et les hommes, Paris : Gallimard.

Daumal, S. (2015). Design d'expérience utilisateur : principes et méthodes UX, Paris : Éditions Eyrolles.

Deterding, S., Dixon, D., Khaled, R. \& Nacke, L. (2014). Du game design au gamefulness : définir la gamification. Sciences du jeu 2, 1-20. Disponible à : https://journals.openedition.org/sdj/287.

Frochot, C., Barberi-Heyob, M., Blanchard-Desce, M., Bolotine, L., Bonneau, S., Jimenez, C. M., Durand, J. O., Lassalle, H. P., Lemercier, G., Mordon, S., Maillard, P., Sol, V., Vever-Bizet, C. \& Vicendo, P. (2015). Thérapie photodynamique : état de l'art et perspectives. L'actualité chimique 397-398. Disponible à : https://hal. archives-ouvertes.fr/hal-01174853.

Genvo, S. (2013). Penser la formation et les évolutions du jeu sur support numérique, Habilitation à diriger des Recherches. Mémoire soutenu à l'Université de Lorraine sous la direction de Jacques Walter. Disponible à : http://www.omnsh.org/sites/default/files/hdr_sg_t1.pdf. 
Goria, S. (2016). Les visualisations de données inspirées par le jeu et la conception par disengamement. Les Cahiers du numérique 12(4), 39-64. Doi :10.3166/lcn.12.4.3964.

Grund, C. \& Schelkle, M. (2016). Developing a serious game for business information visualization, Developing a Serious Game for Business Information Visualization. Twenty-second Americas Conference on Information Systems. San Diego.

Henriot, J. (1969). Le Jeu. Paris : Presses universitaires de France.

Henriot, J. (1989). Sous couleur de jouer. Paris : José Corti.

Huizinga, J. (1938). Homo ludens. Proeve fleener bepaling van het spelelement der cultuur. Haarlem: Tjeenk Willink. [Homo ludens : Essai sur la fonction sociale du jeu, Paris : Gallimard (traduction de 1951)].

Huotari, K. \& Hamari, J. (2017). A definition for gamification: anchoring gamification in the service marketing literature. Electronic Markets 27(1), 21-31.

Kapp, K., Blair, L. \& Mesch, R. (2014). The Gamification of learning and Instruction Fieldbook: Ideas to Practice. San Francisco: Wiley Editions.

Kelley, T. \& Kelley, D. (2016). La confiance créative: tous innovateurs avec le design thinking. Paris : InterEditions.

Kim, W. C. \& Mauborgne, R. (2000). Knowing a winning business idea when you see one. Harvard business review 78(5), 129-138. Doi : 10.1016/S0737-6782(01)000819.

Kirk, A. (2016). Data visualisation: a handbook for data driven design. London : Sage.

Lafourcade, M., Le Brun, N. \& Joubert, A. (2015). Jeux et intelligence collective : résolution de problèmes et acquisition de données sur le Web, London : iSTE Éditions.

Lavigne, M. (2012). Serious games : que devient le plaisir ludique ? Communication présenté au Colloque Ludovia, Ax-Les-Thermes, 27 au 30 août 2012.

Le Béchec, M. (2016). Le territoire comme un graphe. Les Cahiers du numérique 12(4), 131-156.

Morschheuser, B., Hamari, J., Werder, K. \& Abe, J. (2017). How to gamify? A method for designing gamification. Proceedings of the 50th Hawaii International Conference on System Sciences, 1298-1307. Disponible à : http://hdl.handle.net/10125/41308.

Plantin, J.-C. (2014). L'avènement de la carte comme médiation. Questions de communication 25, 309-326. Doi : 10.4000/questionsdecommunication.9050.

Rabardel, P. (1995). Les hommes et les technologies : approche cognitive des instruments contemporains. Paris : Armand Colin.

Ryan, L. (2016). The Visual Imperative: Creating a Visual Culture of Data Discovery. Cambridge MA : Morgan Kaufmann.

Salen, K. \& Zimmerman, E. (2004). Rules of play: Game design fundamentals. London : MIT Press.

Schell, J (2010). L'art du game design. 100 objectifs pour mieux concevoir vos jeux. Paris : Pearson Education France.

Togsverd-Bo, K., Halldin, C., Sandberg, C., Gonzalez, H., Wennberg, A. M., Sørensen, S. S., Wulf, H. C. \& Haedersdal, M. (2017). Photodynamic therapy is more effective than imiquimod for actinic keratosis in organ transplant recipients-a randomized intra-individual controlled trial. British Journal of Dermatology 178(4), 903-909.

Ward, M., Grinstein, G. \& Keim, D. (2015). Interactive Data Visualization: Foundations, Techniques, and Applications. Natik MA : A.K. Peters Ltd. 
Yonnet, P. (1999). Travail, loisir. Temps libre et lien social. Paris : Gallimard.

Zichermann, G. \& Linder, J. (2013), The Gamification Revolution: How Leaders Leverage Game Mechanics to Crush the Competition, New York : McGraw-Hill. 


\section{(c) $(\$)$ \\ BY NC ND Publié sous la licence Creative Commons}

«Attribution - Pas d'Utilisation Commerciale - Pas de Modification 4.0 International» (CC BY-NC-ND) 